\title{
Estimation of Population Mean and Median using Double Robust Truncation based Ranked Set Sampling
}

\author{
Azhar Mehmood Abbasi \\ Department of Statistics, Quaid-i-Azam university, Pakistan \\ abbasiqau2007@yahoo.com \\ Mohammad Yousaf Shad \\ Department of Statistics, Quaid-i-Azam university, Pakistan \\ yousuf@qau.edu.pk
}

\begin{abstract}
In this paper, we propose a double robust truncation based ranked set sampling (DRTBRSS) for estimating population mean and median. It provides unbiased estimator of population mean under symmetric population and its efficiency dominates the efficiency of conventional estimators based on ranked set sampling (RSS), truncation based rank set sampling (TBRSS) and simple random sampling (SRS). Monte Carlo simulation, based on perfect ranking and ranking based on auxiliary variable, is used under symmetric and asymmetric distributions to evaluate effectiveness of the proposed sampling method. Further, a real data set is used to illustrate the proposed sampling design.
\end{abstract}

Subject Classification: MSC2010-62D05

Keywords: Simple random sample, Ranked set sampling, Truncation based ranked set sampling, Double ranked set sampling, Auxiliary variable.

\section{Introduction}

Rank set sampling (RSS) was suggested by McIntyre (1952) for estimating mean pasture and forage yield. Thereafter, many modifications were made in the basic RSS to make it more efficient. For instance, Al-Saleh and Al-Kadiri (2000) suggested double ranked set sampling design for estimating population mean. Samawi et al. (1996) suggested extreme ranked set sampling (ERSS). Al-Omari (2011) introduced double robust extreme ranked set sampling (DRERSS) for efficient estimation of population mean, Al-Nasser (2007) proposed L-ranked set sampling (LRSS) for estimating population mean, Al-Omari and Raqab (2013) introduced truncation based rank set sampling (TBRSS) for estimating population mean and median. Dell and Clutter (1972) showed that even in case of ranking errors, mean estimator based on RSS sampling is at least efficient than its conventional estimator based on SRS. Stokes (1977) presented a simple linear model and showed that an auxiliary variable can be used to rank the study variable. Zamanzade and Al-Omari (2016) introduced neoteric ranked set sampling (NRSS) and compared its efficiency with ordinary RSS and SRS.

In this paper, we proposed a double robust truncation based ranked set sampling (DRTBRSS) for estimating population mean and median. The proposed sampling design provides efficient estimators of population mean and median than conventional estimators based on SRS, RSS and TBRSS. The rest of the paper is organized as follows: In Section 2, RSS, TBRSS, DRSS and the proposed DRTBRSS are described. Estimation of population mean is included in Section 3. Median estimation is considered in Section 
4. DRTBRSS with auxiliary variable is studied in Section 5. Illustration of proposed DRTBRSS with a real data set is explained in Section 6. Finally, concluding remarks are given Section 7.

\section{Sampling Methods}

In this Section we describe the RSS, TBRSS, DRSS and DRTBRSS methods.

\subsection{Rank Set Sampling (RSS)}

RSS can be described as: For selection of $\mathrm{m}$ units, identify $\mathrm{m}^{2}$ units from target population and arrange them into m samples each of size $\mathrm{m}$ and rank the units within each sample with respect to variable of interest by any cost free method. From the ith $(\mathrm{i}=1,2,3, \ldots, \mathrm{m})$ sample, select the $\mathrm{ith}$ smallest ranked unit for actual measurement. The whole procedure can be repeated $r$ times, if needed, to get a RSS sample of size mr.

\subsection{Truncation Based Ranked Set Sampling (TBRSS)}

TBRSS can be described as: draw m simple random samples each of size $\mathrm{m}$ from target population and rank the units within each sample with respect to variable of interest by any cost free method. Define a coefficient $\mathrm{k}=[\alpha \mathrm{m}]$ where $0 \leq \alpha<0.5$ and $[\mathrm{t}]$ is the largest integer less than equal to t. From first k samples, select the smallest rank unit and from the last $\mathrm{k}$ samples select the largest rank units and from the remaining $(\mathrm{m}-2 \mathrm{k})$ samples, select the ith unit of the ith $(i=k+1, k+2, \ldots, m-k)$ sample.

\subsection{Double ranked set sampling (DRSS)}

The DRSS can be described as: Identify $\mathrm{m}^{3}$ units from the target population and divide these units into $\mathrm{m}$ sets each of size $\mathrm{m}^{2}$ units. Apply RSS procedure on each set to obtain $\mathrm{m}$ ranked set samples each of size $\mathrm{m}$. Apply again RSS procedure on $\mathrm{m}$ ranked set samples each of size $m$ to obtain a DRSS of size $m$.

\subsection{The Proposed Sampling Design}

The DRTBRSS is delineated as follows:

Step-1: Identify $\mathrm{m}^{3}$ units from the target population and partition them into $\mathrm{m}$ sets each of size $\mathrm{m}^{2}$ units.

Step-2: Define a coefficient $\mathrm{k}=[\alpha \mathrm{m}]$ where $0 \leq \alpha<0.5$ and $[\mathrm{t}]$ is the largest integer less than equal to $t$.

Step-3: From first k samples of ith set, select the kth smallest ranked units and from the last $\mathrm{k}$ samples of ith set, select the $(\mathrm{m}-\mathrm{k}+1)$ th smallest ranked units. From the remaining $(\mathrm{m}-2 \mathrm{k})$ samples of ith $(\mathrm{i}=1,2,3, \ldots, \mathrm{m})$ set, select the $\mathrm{jth}$ $(\mathrm{j}=\mathrm{k}+1, \mathrm{k}+2, \ldots, \mathrm{m}-\mathrm{k})$ sample. This step finally yields $\mathrm{m}$ samples each of size m.

Step-4: From $m$ samples each of size $m$ obtained in step-3 above, select from first $\mathrm{k}$ samples the kth smallest ranked units and from the last $\mathrm{k}$ samples the 
$(\mathrm{m}-\mathrm{k}+1)$ th smallest ranked units. From the remaining $(\mathrm{m}-2 \mathrm{k})$ samples, select the jth unit of the $\mathrm{jth}(\mathrm{j}=\mathrm{k}+1, \mathrm{k}+2, \ldots, \mathrm{m}-\mathrm{k})$ sample to get a DRTBRSS of size $\mathrm{m}$.

This completes one cycle for selection of a sample of size m units under DRTBRSS. The above steps1-4 can be repeated $r$ times, if needed, to obtain a sample of size mr units. It may be noted that the above proposed sampling design is called robust in the sense that it also works better than ordinary TBRSS when underlying population is skewed.

Example 1. Suppose $\mathrm{m}=5$ and $\mathrm{k}=1,2$. In this case we need $\mathrm{m}^{3}=125$ units and layout these units in $\mathrm{m}=5$ sets each of size $\mathrm{m}^{2}=25$. Let $\mathrm{X}_{\mathrm{i}(\mathrm{j}: \mathrm{m})}^{1}$ denotes $\mathrm{jth}$ ranked unit of the ith sample of size $\mathrm{m}$ in 1 th set. Then, after ranking the units with respect to variable of interest by any cost free method, we have

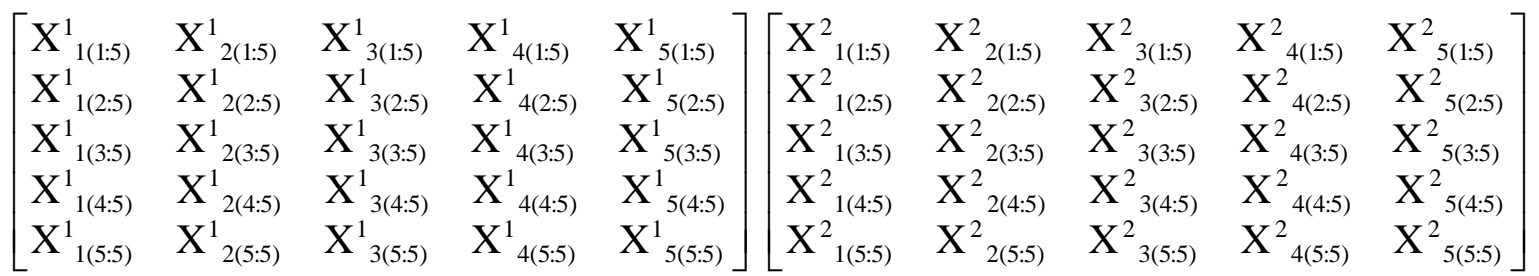

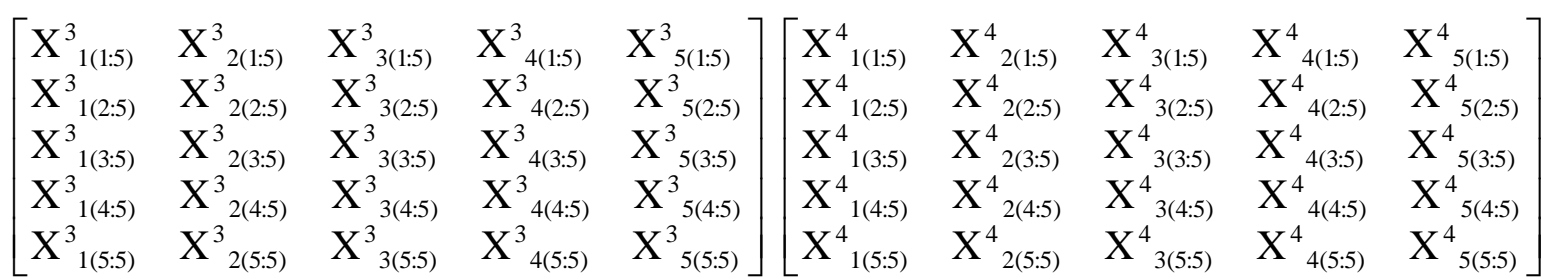

and

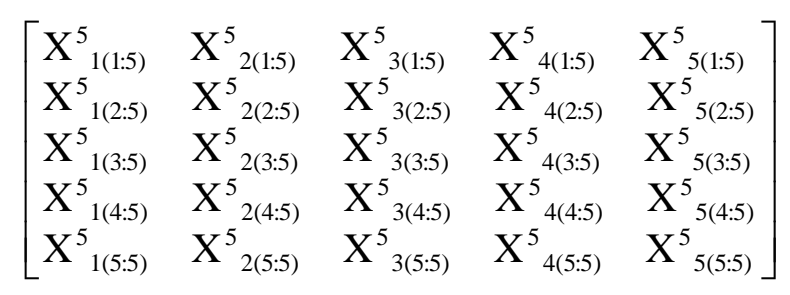

Case 1: For $\mathrm{k}=1$

From first $\mathrm{k}=1$ sample of ith set, select kth smallest ranked unit and from last $\mathrm{k}=1$ sample of ith set, select $(\mathrm{m}-\mathrm{k}+1)$ th smallest ranked units. From the remaining $\mathrm{m}-2 \mathrm{k}=3$ samples of $i$ th $(i=1,2,3, \ldots, 5)$ set, select $j$ th smallest unit of $j$ th $(j=2,3,4)$ sample. Then, we have following 5 samples each of size $m=5$.

$$
\begin{gathered}
S_{1}=\left\{X_{1(1: 5)}^{1}, X_{2(2: 5)}^{1}, X_{3(3: 5)}^{1}, X_{4(4: 5)}^{1}, X_{5(5: 5)}^{1}\right\}, S_{2}=\left\{X_{1(1: 5)}^{2}, X_{2(2: 5)}^{2}, X_{3(3: 5)}^{2}, X_{4(4: 5)}^{2}, X^{2}{ }_{5(5: 5)}\right\} \\
S_{3}=\left\{X_{1(1: 5)}^{3}, X_{2(2: 5)}^{3}, X_{3(3: 5)}^{3}, X_{4(4: 5)}^{3}, X_{5(5: 5)}^{3}\right\}, S_{4}=\left\{X_{1(1: 5)}^{4}, X_{2(2: 5)}^{4}, X_{3(3: 5)}^{4}, X_{4(4: 5)}^{4}, X^{4}{ }_{5(5: 5)}\right\} \\
S_{5}=\left\{X_{1(1: 5)}^{5}, X^{5}{ }_{2(2: 5)}, X_{3(3: 5)}^{5}, X_{4(4: 5)}^{5}, X_{5(5: 5)}^{5}\right\}
\end{gathered}
$$


Let $Y_{1}$ be the kth smallest unit of $S_{1}, Y_{2}$ be the $(k+1)$ th smallest unit of $S_{2}, Y_{3}$ be the $(k+2)$ th smallest unit of $S_{3}, Y_{4}$ be the $(m-k)$ th smallest unit of $S_{4}$ and $Y_{5}$ be the $(\mathrm{m}-\mathrm{k}+1)$ th smallest unit of $\mathrm{S}_{5}$ then $\left\{\mathrm{Y}_{1}, \mathrm{Y}_{2}, \mathrm{Y}_{3}, \mathrm{Y}_{4}, \mathrm{Y}_{5}\right\}$, where $\mathrm{Y}_{\mathrm{i}}$ is actually ith smallest ranked unit of $S_{i}(i=1,2,3, \ldots, 5)$, is DRTBRSS of size 5 .

Case 2: For $\mathrm{k}=2$

From first $\mathrm{k}=2$ samples of ith set, select kth smallest ranked unit and from last $\mathrm{k}=2$ samples of ith set, select $(\mathrm{m}-\mathrm{k}+1)$ th smallest ranked units. From the remaining $\mathrm{m}-2 \mathrm{k}=1$ samples of $\mathrm{ith}(\mathrm{i}=1,2,3, \ldots, 5)$ set, select $\mathrm{jth}$ smallest unit of $\mathrm{jth}(\mathrm{j}=3)$ sample. Then, we have following 5 samples each of size $m=5$.

$$
\begin{gathered}
S_{1}=\left\{X_{1(2: 5)}^{1}, X_{2(2: 5)}^{1}, X_{3(3: 5)}^{1}, X_{4(4: 5)}^{1}, X_{5(4: 5)}^{1}\right\}, S_{2}=\left\{X_{1(2: 5)}^{2}, X_{2(2: 5)}^{2}, X_{3(3: 5)}^{2}, X_{4(4: 5)}^{2}, X_{5(4: 5)}^{2}\right\} \\
S_{3}=\left\{X_{1(2: 5)}^{3}, X_{2(2: 5)}^{3}, X_{3(3: 5)}^{3}, X_{4(4: 5)}^{3}, X^{3}{ }_{5(4: 5)}\right\}, S_{4}=\left\{X_{1(2: 5)}^{4}, X_{2(2: 5)}^{4}, X_{3(3: 5)}^{4}, X_{4(4: 5)}^{4}, X_{5(4: 5)}^{4}\right\} \\
S_{5}=\left\{X_{1(2: 5)}^{5}, X_{2(2: 5)}^{5}, X_{3(3: 5)}^{5}, X_{4(4: 5)}^{5}, X_{5(4: 5)}^{5}\right\}
\end{gathered}
$$

Let $Y_{1}$ be the kth smallest unit of $S_{1}, Y_{2}$ be the kth smallest unit of $S_{2}, Y_{3}$ be the $(k+1)$ th smallest unit of $S_{3}, Y_{4}$ be the $(m-k+1)$ th smallest unit of $S_{4}$ and $Y_{5}$ be the $(\mathrm{m}-\mathrm{k}+1)$ th smallest unit of $\mathrm{S}_{5}$ then $\left\{\mathrm{Y}_{1}, \mathrm{Y}_{2}, \mathrm{Y}_{3}, \mathrm{Y}_{4}, \mathrm{Y}_{5}\right\}$, where $\mathrm{Y}_{1}, \mathrm{Y}_{2}$ are actually second smallest ranked units of $S_{i}(i=1,2), Y_{3}$ is third smallest ranked unit of $S_{3}$ and $\mathrm{Y}_{4}, \mathrm{Y}_{5}$ are fourth smallest ranked units of $\mathrm{S}_{\mathrm{j}}(\mathrm{j}=4,5)$, is DRTBRSS of size 5 .

\section{Estimation of population mean}

Let the variable of interest $X$ has probability density function (pdf) $f(x)$ and cumulative distribution function $F(x)$ with mean $\mu$ and variance $\sigma^{2}$. Let $X_{1}, X_{2}, X_{3}, \ldots, X_{m}$ be a SRS of size $\mathrm{m}$ from $\mathrm{f}(\mathrm{x})$. The SRS estimator of population mean $\mu$ if sampling is repeated $\mathrm{r}$ times, is defined as $\overline{\mathrm{X}}_{\mathrm{SRS}}=\frac{1}{\mathrm{mr}} \sum_{\mathrm{j}=1}^{\mathrm{r}} \sum_{\mathrm{i}=1}^{\mathrm{m}} \mathrm{X}_{\mathrm{ij}}$ with its variance $\sigma_{\overline{\mathrm{X}}_{\mathrm{SRS}}^{2}}^{2}=\frac{\sigma_{\mathrm{X}}^{2}}{\mathrm{mr}}$. Let $\mathrm{X}_{\mathrm{i} 1}, \mathrm{X}_{\mathrm{i} 2}, \mathrm{X}_{\mathrm{i} 3}, \ldots, \mathrm{X}_{\mathrm{im}} \quad(\mathrm{i}=1,2,3, \ldots, \mathrm{m})$ denote $\mathrm{m}$ SRS each of size $\mathrm{m}$. Suppose, $\mathrm{X}_{\mathrm{i}(1: \mathrm{m})}, \mathrm{X}_{\mathrm{i}(2: \mathrm{m})}, \ldots, \mathrm{X}_{\mathrm{i}(\mathrm{m}: \mathrm{m})}$ denote order statistics of the ith sample.Then, $\mathrm{X}_{1(1: \mathrm{m})}, \mathrm{X}_{2(2: \mathrm{m})}, \ldots, \mathrm{X}_{\mathrm{m}(\mathrm{m}: \mathrm{m})}$ is called $\mathrm{RSS}$ of size $\mathrm{m}$.

Let $\mathrm{g}_{(\mathrm{i}: \mathrm{m})}(\mathrm{x})$ be pdf of ith order statistic i.e. $\mathrm{X}_{(\mathrm{i}: \mathrm{m})}(\mathrm{i}=1,2,3, \ldots, \mathrm{m})$, then it can be shown that:

$$
\mathrm{g}_{(\mathrm{i}: \mathrm{m})}(\mathrm{x})=\mathrm{m}\left(\begin{array}{c}
\mathrm{m}-1 \\
\mathrm{i}-1
\end{array}\right)(\mathrm{F}(\mathrm{x}))^{\mathrm{i}-1}(1-\mathrm{F}(\mathrm{x}))^{\mathrm{m}-\mathrm{i}} \mathrm{f}(\mathrm{x})-\infty<\mathrm{x}<\infty
$$


The mean and variance of $\mathrm{X}_{(\mathrm{i}: \mathrm{m})}$ respectively are given by

$$
\mu_{(\mathrm{i}: \mathrm{m})}=\int_{-\infty}^{\infty} \mathrm{xg}_{(\mathrm{i}: \mathrm{m})}(\mathrm{x}) \mathrm{dx}
$$

and $\quad \sigma_{(\mathrm{i}: \mathrm{m})}^{2}=\int_{-\infty}^{\infty}\left(\mathrm{x}-\mu_{(\mathrm{i}: \mathrm{m})}\right)^{2} \mathrm{~g}_{(\mathrm{i}: \mathrm{m})}(\mathrm{x}) \mathrm{dx}$

for detail see David and Nagaraja (2003). The RSS estimator of population mean, say $\mu$, is defined as

$$
\bar{X}_{\mathrm{RSS}}=\frac{1}{\mathrm{mr}} \sum_{\mathrm{j}=1 \mathrm{i}=1}^{\mathrm{r}} \sum_{\mathrm{i}(\mathrm{i}: \mathrm{m}) \mathrm{j}}
$$

and its variance is given by

$$
\operatorname{Var}\left(\bar{X}_{\mathrm{RSS}}\right)=\frac{1}{\mathrm{~m}^{2} \mathrm{r}} \sum_{\mathrm{i}=1}^{\mathrm{m}} \sigma_{(\mathrm{i}: \mathrm{m})}^{2}=\frac{\sigma_{\mathrm{X}}^{2}}{\mathrm{mr}}-\frac{1}{\mathrm{~m}^{2} \mathrm{r}} \sum_{\mathrm{i}=1}^{\mathrm{m}}\left(\mu_{(\mathrm{i}: \mathrm{m})}-\mu_{\mathrm{X}}\right)^{2}
$$

\subsection{Estimation of population mean using TBRSS}

Let $\mathrm{X}_{\mathrm{i} 1}, \mathrm{X}_{\mathrm{i} 2}, \mathrm{X}_{\mathrm{i} 3}, \ldots, \mathrm{X}_{\mathrm{im}}$ be the ith $(\mathrm{i}=1,2,3, \ldots, \mathrm{m})$ SRS of size $\mathrm{m}$, then the TBRSS estimator of population mean $\mu$ and its variance are defined as The TBRSS estimator of population mean is given by

$$
\bar{X}_{\text {TBRSS }}=\frac{1}{m r}\left(\sum_{\mathrm{j}=1 \mathrm{i}=1}^{\mathrm{r}} \sum_{\mathrm{i}(1: \mathrm{m}) \mathrm{j}}+\sum_{\mathrm{j}=1 \mathrm{i}=\mathrm{k}+1}^{\mathrm{r}} \sum_{\mathrm{i}(\mathrm{i}: \mathrm{m}) \mathrm{j}}+\sum_{\mathrm{j}=1 \mathrm{i}=\mathrm{m}-\mathrm{k}+1}^{\mathrm{r}} \sum_{\mathrm{i}(\mathrm{m}: \mathrm{m}) \mathrm{j}}^{\mathrm{m}}\right)
$$

with its variance

$$
\operatorname{Var}\left(\bar{X}_{\mathrm{TBRSS}}\right)=\frac{1}{\mathrm{~m}^{2} \mathrm{r}}\left\{\mathrm{k}\left(\sigma_{(\mathrm{l}: \mathrm{m})}^{2}+\sigma_{(\mathrm{m}: \mathrm{m})}^{2}\right)+\sum_{\mathrm{i}=\mathrm{k}+1}^{\mathrm{m}-\mathrm{k}} \sigma_{(\mathrm{i}: \mathrm{m})}^{2}\right\}
$$

\subsection{Estimation of population mean using DRTBRSS}

Let $\quad X_{1(k: m)}^{\cdot}, X_{2(k: m)}^{\cdot}, \ldots, X_{k(k: m)}^{\cdot}, X_{(k+1)((k+1): m)}^{\cdot}, \ldots, X_{(m-k)(m-k: m)}^{\cdot}, \quad X_{(m-k+1)((m-k+1): m)}^{\cdot}, \ldots$, $\mathrm{X}_{\mathrm{m}(\mathrm{m}-\mathrm{k}+\mathrm{l}: \mathrm{m})}$ be a DRTBRSS of size $\mathrm{m}$. The DRTBRSS estimator of population mean $\mu$ is defined as

$$
\bar{X}_{\text {DRTBRSS }}=\frac{1}{m r}\left(\sum_{j=1 i=1}^{r} \sum_{i(k: m) j}^{k}+\sum_{j=1 i=k+1}^{r} \sum_{i(i: m) j}^{m-k}+\sum_{j=1 i=m-k+1}^{r} \sum_{i(m-k+1: m) j}^{m}\right)
$$

with variance

$$
\operatorname{Var}\left(\overline{\mathrm{X}}_{\text {DRTBRSS }}\right)=\frac{1}{\mathrm{~m}^{2} \mathrm{r}}\left\{\mathrm{k}\left(\sigma_{(\mathrm{k}: \mathrm{m})}^{2 \cdot}+\sigma_{(\mathrm{m}-\mathrm{k}+1 \mathrm{~m})}^{2 \cdot}\right)+\sum_{\mathrm{i}=\mathrm{k}+1}^{\mathrm{m}-\mathrm{k}} \sigma_{(\mathrm{i}: \mathrm{m})}^{2 \cdot}\right\}
$$

Remarks 1: It may be noted that the DRTBRSS estimator given by $\mathrm{Eq}(6)$ reduces to the mean estimator based on DRSS for $\mathrm{k}=0,1$, which shows that DRSS is a special case of DRTBRSS.

If the underlying distribution is symmetric about its mean, say $\mu$, we have $\sigma_{(\mathrm{i}: \mathrm{m})}^{2 \cdot}=\sigma_{(\mathrm{m}-\mathrm{i}+1 \mathrm{l})}^{2 \cdot}$, for details see David and Nagaraja (2003). Therefore, $\operatorname{Eq}(7)$ further reduces to

$$
\operatorname{Var}\left(\overline{\mathrm{X}}_{\mathrm{DRTBRSS}}\right)=\frac{1}{\mathrm{~m}^{2} \mathrm{r}}\left\{2 \mathrm{k} \sigma_{(\mathrm{k}: \mathrm{m})}^{2 \cdot}+\sum_{\mathrm{i}=\mathrm{k}+1}^{\mathrm{m}-\mathrm{k}} \sigma_{(\mathrm{i}: \mathrm{m})}^{2 \cdot}\right\}
$$


If the underlying distribution is asymmetric, the mean square error (MSE) of the proposed estimator is given by

$$
\operatorname{Var}\left(\overline{\mathrm{X}}_{\mathrm{DRTBRSS}}\right)=\frac{1}{\mathrm{~m}^{2} \mathrm{r}}\left\{\mathrm{k}\left(\sigma_{(\mathrm{k}: \mathrm{m})}^{2 \cdot}+\sigma_{(\mathrm{m}-\mathrm{k}+\mathrm{l}: \mathrm{m})}^{2 \cdot}\right)+\sum_{\mathrm{i}=\mathrm{k}+1}^{\mathrm{m}-\mathrm{k}} \sigma_{(\mathrm{i}: \mathrm{m})}^{2 \cdot}\right\}+(\text { Bias })^{2}
$$

Where Bias $=\mathrm{E}\left(\overline{\mathrm{X}}_{\mathrm{DRTBRSS}}-\mu\right)$. It may be noted that MSE and Bias of any estimator $\mathrm{T}(\cdot)$ of population parameter $\mu$ are defined as $\operatorname{MSE}(\mathrm{T}(\cdot))=\operatorname{Var}(\mathrm{T}(\cdot))+(\operatorname{Bias})^{2}$ and $\operatorname{Bias}=\mathrm{E}(\mathrm{T}(\cdot)-\mu)$

For symmetric distribution, the relative efficiency of the TBRSS and DRTBRSS with respect to SRS is defined as

$$
\operatorname{RE}\left(\overline{\mathrm{X}}_{\mathrm{J}}, \overline{\mathrm{X}}_{\mathrm{SRS}}\right)=\frac{\operatorname{Var}\left(\overline{\mathrm{X}}_{\mathrm{SRS}}\right)}{\operatorname{Var}\left(\overline{\mathrm{X}}_{\mathrm{J}}\right)} \mathrm{J}=\mathrm{TBRSS}, \mathrm{DRTBRSS}
$$

For asymmetric distribution, the RE of TBRSS and DRTBRSS with respect to SRS is given by

$$
\operatorname{RE}\left(\bar{X}_{\mathrm{J}}, \overline{\mathrm{X}}_{\mathrm{SRS}}\right)=\frac{\operatorname{Var}\left(\overline{\mathrm{X}}_{\mathrm{SRS}}\right)}{\operatorname{MSE}\left(\overline{\mathrm{X}}_{\mathrm{J}}\right)} \quad \mathrm{J}=\mathrm{TBRSS}, \mathrm{DRTBRSS}
$$

Lemma-1: If the underlying distribution is symmetric, $\overline{\mathrm{X}}_{\text {DRTBRSS }}$ is unbiased estimator of population mean $\mu$.

Proof: From $\mathrm{Eq}(6)$, we have

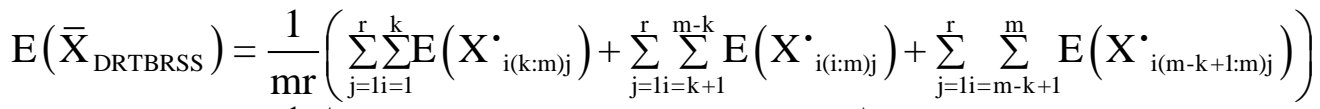

$$
\begin{aligned}
& =\frac{1}{\mathrm{~m}}\left(\mathrm{k} \mu \dot{ }_{(\mathrm{k}: \mathrm{m})}+\sum_{\mathrm{i}=\mathrm{k}+1}^{\mathrm{m}-\mathrm{k}} \mu \dot{(i: m)}+\mathrm{k} \mu \dot{(m-\mathrm{m}+1: \mathrm{m})}\right) \\
& =\frac{1}{\mathrm{~m}}\left(\mathrm{k}\left(\mu^{\cdot}{ }_{(\mathrm{k}: \mathrm{m})}+\mu^{\cdot}{ }_{(\mathrm{m}-\mathrm{k}+1: \mathrm{m})}\right)+\sum_{\mathrm{i}=\mathrm{k}+1}^{\mathrm{m}-\mathrm{k}} \mu^{\cdot}{ }_{(\mathrm{i}: \mathrm{m})}\right)
\end{aligned}
$$

For symmetric distribution, we have $\mu_{\mathrm{i}}^{\cdot}+\mu_{\mathrm{m}-\mathrm{i}+1}^{\cdot}=2 \mu$. Further, it is easy to write

$$
\begin{aligned}
& \sum_{\mathrm{i}=1}^{\mathrm{m}} \mu_{(\mathrm{i}: \mathrm{m})}=\mathrm{m} \mu=\sum_{\mathrm{i}=1}^{\mathrm{k}} \mu_{(\mathrm{i}: \mathrm{m})}+\sum_{\mathrm{i}=\mathrm{k}+1}^{\mathrm{m}-\mathrm{k}} \mu_{(\mathrm{i}: \mathrm{m})}+\sum_{\mathrm{i}=\mathrm{m}-\mathrm{k}+1}^{\mathrm{m}} \mu_{(\mathrm{i}: \mathrm{m})} \cdot \\
& \therefore \mathrm{E}\left(\overline{\mathrm{X}}_{\text {DRTBRSS }}\right)=\frac{1}{\mathrm{~m}}\left(2 \mathrm{k} \mu+\mathrm{mu}-\sum_{\mathrm{i}=1}^{\mathrm{k}} \mu_{(\mathrm{i}: \mathrm{m})}-\sum_{\mathrm{i}=\mathrm{m}-\mathrm{k}+1}^{\mathrm{m}} \mu_{(\mathrm{i}: \mathrm{m})}\right) \\
& =\frac{1}{\mathrm{~m}}(2 \mathrm{k} \mu+\mathrm{mu}-2 \mathrm{k} \mu) \\
& =\mu
\end{aligned}
$$

Lemma-2: For symmetric distribution, DRTBRSS is more efficient than SRS.

Proof: Consider the $\mathrm{Eq}(8)$

$$
\operatorname{Var}\left(\overline{\mathrm{X}}_{\text {DRTBRSS }}\right)=\frac{1}{\mathrm{~m}^{2} \mathrm{r}}\left\{2 \mathrm{k} \sigma_{(\mathrm{k}: \mathrm{m})}^{2 \cdot}+\sum_{\mathrm{i}=\mathrm{k}+1}^{\mathrm{m}-\mathrm{k}} \sigma_{(\mathrm{i}: \mathrm{m})}^{2 \cdot}\right\}
$$


Note that $\sum_{\mathrm{i}=1}^{\mathrm{m}} \sigma_{(\mathrm{i}: \mathrm{m})}^{2 \cdot}=\sum_{\mathrm{i}=1}^{\mathrm{k}} \sigma_{(\mathrm{i}: \mathrm{m})}^{2 \cdot}+\sum_{\mathrm{i}=\mathrm{k}+1}^{\mathrm{t}-\mathrm{k}} \sigma_{(\mathrm{i}: \mathrm{m})}^{2 \cdot}+\sum_{\mathrm{i}=\mathrm{t}-\mathrm{k}+1}^{\mathrm{t}} \sigma_{(\mathrm{i}: \mathrm{m})}^{2 \cdot}$.

$$
\begin{aligned}
\therefore \operatorname{Var}\left(\bar{X}_{\text {DRTBRSS }}\right) & =\frac{1}{m^{2} \mathrm{r}}\left(2 \mathrm{k} \sigma_{(\mathrm{k}: \mathrm{m})}^{2 \cdot}+\sum_{\mathrm{i}=1}^{\mathrm{m}} \sigma_{(\mathrm{i}: \mathrm{m})}^{2 \cdot}-\sum_{\mathrm{i}=1}^{\mathrm{k}} \sigma_{(\mathrm{i}: \mathrm{m})}^{2 \cdot}-\sum_{\mathrm{i}=\mathrm{m}-\mathrm{k}+1}^{\mathrm{m}} \sigma_{(\mathrm{i}: \mathrm{m})}^{2 \cdot}\right) \\
& =\frac{1}{\mathrm{~m}^{2} \mathrm{r}}\left(2 \mathrm{k} \sigma_{(\mathrm{k}: \mathrm{m})}^{2 \cdot}+\sum_{\mathrm{i}=1}^{\mathrm{m}} \sigma_{(\mathrm{i}: \mathrm{m})}^{2 \cdot}-2 \sum_{\mathrm{i}=1}^{\mathrm{k}} \sigma_{(\mathrm{i}: \mathrm{m})}^{2 \cdot}\right) \\
& =\frac{1}{\mathrm{~m}^{2} \mathrm{r}} \sum_{\mathrm{i}=1}^{\mathrm{m}} \sigma_{(\mathrm{i}: \mathrm{m})}^{2 \cdot}-\frac{2}{\mathrm{~m}^{2} \mathrm{r}}\left(\sum_{\mathrm{i}=1}^{\mathrm{k}} \sigma_{(\mathrm{i}: \mathrm{m})}^{2 \cdot}-\mathrm{k} \sigma_{(\mathrm{k}: \mathrm{m})}^{2 \cdot}\right)
\end{aligned}
$$

Here, the first term on the right hand side is variance of population mean estimator based on DRSS. Further, we know the relationship between variances of mean estimators based on SRS and DRSS as given by

$$
\begin{aligned}
& \sigma_{\mathrm{X}}^{2}=\frac{1}{\mathrm{~m}} \sum_{\mathrm{i}=1}^{\mathrm{m}} \sigma_{(\mathrm{i}: \mathrm{m})}^{2 \cdot}+\frac{1}{\mathrm{~m}} \sum_{\mathrm{i}=1}^{\mathrm{m}}\left(\mu_{(\mathrm{i}: \mathrm{m})}^{\cdot}-\mu\right)^{2} \\
& \operatorname{Var}\left(\bar{X}_{\text {DRTBRSS }}\right)=\frac{1}{\mathrm{mr}} \sigma_{\mathrm{X}}^{2}-\frac{2}{\mathrm{~m}^{2} \mathrm{r}}\left(\sum_{\mathrm{i}=1}^{\mathrm{k}}{\sigma_{(\mathrm{i}: \mathrm{m})}^{2 \cdot}}^{\cdot} \mathrm{k} \sigma_{(\mathrm{k}: \mathrm{m})}^{2 \cdot}+\frac{1}{2} \sum_{\mathrm{i}=1}^{\mathrm{m}}\left(\mu \cdot{ }_{(\mathrm{i}: \mathrm{m})}-\mu\right)^{2}\right)
\end{aligned}
$$

Note that for symmetric distribution the variance decreases as i increases with minimum value occurs at $\mathrm{i}=\left[\frac{\mathrm{m}+1}{2}\right]$, which means $\sigma_{(\mathrm{i}: \mathrm{m})}^{2 \cdot} \leq \sigma_{(\mathrm{j}: \mathrm{m})}^{2 \cdot} \forall \mathrm{i} \geq \mathrm{j}$ and $\mathrm{j} \leq\left[\frac{\mathrm{m}+1}{2}\right]$, for details see Al-Nasser (2007). So the second term becomes positive and $\operatorname{Var}\left(\overline{\mathrm{X}}_{\mathrm{SRS}}\right)=\frac{\sigma^{2}}{\mathrm{mr}}$. This completes the proof.

Lemma-3: if underlying distribution is symmetric, the $\bar{X}_{\text {DRTBRSS }}$ is more efficient than $\overline{\mathrm{X}}_{\mathrm{RSS}}$ if

$$
\sum_{\mathrm{i}=1}^{\mathrm{m}} \sigma_{(\mathrm{i}: \mathrm{m})}^{2} \geq 2 \mathrm{k} \sigma_{(\mathrm{k}: \mathrm{m})}^{2 \cdot}+\sum_{\mathrm{i}=\mathrm{k}+1}^{\mathrm{m}-\mathrm{k}} \sigma_{(\mathrm{i}: \mathrm{m})}^{2 \cdot}
$$

Proof: $\bar{X}_{\text {DRTBRSS }}$ will be more efficient than $\bar{X}_{R S S}$ if $\operatorname{Var}\left(\bar{X}_{R S S}\right) \geq \operatorname{Var}\left(\bar{X}_{\text {DRTBRSS }}\right)$ i.e.

$$
\begin{aligned}
& \frac{1}{\mathrm{~m}^{2} \mathrm{r}} \sum_{\mathrm{i}=1}^{\mathrm{m}} \sigma_{(\mathrm{i}: \mathrm{m})}^{2} \geq \frac{1}{\mathrm{~m}^{2} \mathrm{r}}\left\{2 \mathrm{k} \sigma_{(\mathrm{k}: \mathrm{m})}^{2 \cdot}+\sum_{\mathrm{i}=\mathrm{k}+1}^{\mathrm{m}-\mathrm{k}} \sigma_{(\mathrm{i}: \mathrm{m})}^{2 \cdot}\right\} \\
& \text { or } \quad \sum_{\mathrm{i}=1}^{\mathrm{m}} \sigma_{(\mathrm{i}: \mathrm{m})}^{2} \geq 2 \mathrm{k} \sigma_{(\mathrm{k}: \mathrm{m})}^{2 \cdot}+\sum_{\mathrm{i}=\mathrm{k}+1}^{\mathrm{m}-\mathrm{k}} \sigma_{(\mathrm{i}: \mathrm{m})}^{2 \cdot}
\end{aligned}
$$

Which completes the proof.

Lemma-4: If underlying distribution is symmetric, the $\bar{X}_{\text {DRTBRSS }}$ is more efficient than $\overline{\mathrm{X}}_{\mathrm{TBRSS}}$ if

$$
2 \mathrm{k}\left(\sigma_{(1: \mathrm{m})}^{2}-\sigma_{(\mathrm{k}: \mathrm{m})}^{2 \cdot}\right) \geq \sum_{\mathrm{i}=\mathrm{k}+1}^{\mathrm{m}-\mathrm{k}} \sigma_{(\mathrm{i}: \mathrm{m})}^{2 \cdot}-\sum_{\mathrm{i}=\mathrm{k}+1}^{\mathrm{m}-\mathrm{k}} \sigma_{(\mathrm{i}: \mathrm{m})}^{2}
$$


Proof: $\bar{X}_{\text {DRTBRSS }}$ will be more efficient than $\bar{X}_{\text {TBRSS }}$ if $\operatorname{Var}\left(\bar{X}_{\text {TBRSS }}\right) \geq \operatorname{Var}\left(\bar{X}_{\text {DRTBRSS }}\right)$ i.e.

$$
\begin{aligned}
& \frac{1}{\mathrm{~m}^{2} \mathrm{r}}\left\{\mathrm{k}\left(\sigma_{(1: \mathrm{m})}^{2}+\sigma_{(\mathrm{m}: \mathrm{m})}^{2}\right)+\sum_{\mathrm{i}=\mathrm{k}+1}^{\mathrm{m}-\mathrm{k}} \sigma_{(\mathrm{i}: \mathrm{m})}^{2}\right\} \geq \frac{1}{\mathrm{~m}^{2} \mathrm{r}}\left\{2 \mathrm{k} \sigma_{(\mathrm{k}: \mathrm{m})}^{2 \cdot}+\sum_{\mathrm{i}=\mathrm{k}+1}^{\mathrm{m}-\mathrm{k}} \sigma_{(\mathrm{i}: \mathrm{m})}^{2 \cdot}\right\} \\
& 2 \mathrm{k} \sigma_{(1: \mathrm{m})}^{2}+\sum_{\mathrm{i}=\mathrm{k}+1}^{\mathrm{m}-\mathrm{k}} \sigma_{(\mathrm{i}: \mathrm{m})}^{2} \geq 2 \mathrm{k} \sigma_{(\mathrm{k}: \mathrm{m})}^{2 \cdot}+\sum_{\mathrm{i}=\mathrm{k}+1}^{\mathrm{m}-\mathrm{k}} \sigma_{(\mathrm{i}: \mathrm{m})}^{2 \cdot} \quad \because \sigma_{\mathrm{i}}^{2}=\sigma_{\mathrm{m}-\mathrm{i}+1}^{2}
\end{aligned}
$$

or

$$
2 \mathrm{k}\left(\sigma_{(1: \mathrm{m})}^{2}-\sigma_{(\mathrm{k}: \mathrm{m})}^{2 \cdot}\right) \geq \sum_{\mathrm{i}=\mathrm{k}+1}^{\mathrm{m}-\mathrm{k}} \sigma_{(\mathrm{i}: \mathrm{m})}^{2 \cdot}-\sum_{\mathrm{i}=\mathrm{k}+1}^{\mathrm{m}-\mathrm{k}} \sigma_{(\mathrm{i}: \mathrm{m})}^{2}
$$

Which completes the proof.

The relative efficiencies (REs) of the considered sampling designs are calculated using Eqs(10) and (11) against different sample sizes for both symmetric and asymmetric distributions and reported in Tables 1-2. we also calculated absolute bias and put it in parenthesis below the respective REs. The simulated mean, absolute bias and MSE based on 40,000 replications are defined as

$$
\begin{aligned}
& \hat{\mu}_{\mathrm{S}}=\frac{1}{40000} \sum_{\mathrm{i}=1}^{40000} \hat{\mu}_{\mathrm{S}, \mathrm{i}} \quad \operatorname{Abs}\left(\operatorname{bias}\left(\hat{\mu}_{\mathrm{S}}\right)\right)=\frac{1}{40000} \sum_{\mathrm{i}=1}^{40000}\left|\hat{\mu}_{\mathrm{S}, \mathrm{i}}-\mu\right| \\
& \operatorname{MSE}\left(\hat{\mu}_{\mathrm{S}}\right)=\frac{1}{40000} \sum_{\mathrm{i}=1}^{40000}\left(\hat{\mu}_{\mathrm{S}, \mathrm{i}}-\mu\right)^{2} \quad \mathrm{~S}=\mathrm{SRS}, \mathrm{TBRSS}, \mathrm{DRTBRSS}
\end{aligned}
$$

\begin{tabular}{|c|c|c|c|c|c|c|c|c|}
\hline \multirow[b]{3}{*}{ Distribution } & \multicolumn{4}{|c|}{$\mathrm{m}=5$} & \multicolumn{4}{|c|}{$\mathrm{m}=6$} \\
\hline & RSS & TBRSS & DRSS & DRTBRSS & RSS & TBRSS & DRSS & DRTBRSS \\
\hline & $\mathrm{k}=0,1$ & $\mathrm{k}=2$ & $\mathrm{k}=0,1$ & $\mathrm{k}=2$ & $\mathrm{k}=0,1$ & $\mathrm{k}=2$ & $\mathrm{k}=0,1$ & $\mathrm{k}=2$ \\
\hline \multirow[t]{2}{*}{ Uniform $(0,1)$} & 2.9941 & 3.6197 & 5.7198 & 5.5620 & 3.5351 & 3.9994 & 7.1813 & 7.0750 \\
\hline & $(0.002)$ & $(0.001)$ & $(0.001)$ & $(0.040)$ & $(0.000)$ & $(0.001)$ & $(0.000)$ & $(0.001)$ \\
\hline \multirow[t]{2}{*}{$\operatorname{Normal}(0,1)$} & 2.7856 & 2.4097 & 4.4211 & 8.3305 & 3.1801 & 2.7459 & 5.3741 & 10.1156 \\
\hline & $(0.002)$ & $(0.002)$ & $(0.001)$ & $(0.095)$ & $(0.001)$ & $(0.001)$ & $(0.002)$ & $(0.003)$ \\
\hline \multirow[t]{2}{*}{ Logistic $(0,1)$} & 2.5802 & 2.0250 & 3.7911 & 10.2189 & 2.9121 & 2.2734 & 4.4673 & 11.5800 \\
\hline & $(0.003)$ & $(0.004)$ & $(0.003)$ & $(0.150)$ & $(0.002)$ & $(0.002)$ & $(0.001)$ & $(0.004)$ \\
\hline \multirow[t]{2}{*}{ Exponential(1) } & 2.1787 & 1.2762 & 3.0175 & 2.8089 & 2.4741 & 1.5024 & 3.4530 & 2.8905 \\
\hline & $(0.002)$ & $(0.154)$ & $(0.001)$ & $(0.232)$ & $(0.000)$ & $(0.133)$ & $(0.000)$ & $(0.210)$ \\
\hline \multirow[t]{2}{*}{ Weibull(1,3) } & 2.1641 & 1.3218 & 3.0266 & 2.8039 & 2.4107 & 1.4858 & 3.5638 & 2.7948 \\
\hline & $(0.003)$ & $(0.443)$ & $(0.002)$ & $(0.695)$ & $(0.007)$ & $(0.408)$ & $(0.001)$ & $(0.634)$ \\
\hline \multirow[t]{2}{*}{$\operatorname{Gamma}(2,3)$} & 2.4208 & 1.6547 & 3.6064 & 3.9052 & 2.7271 & 1.8760 & 4.1140 & 4.1092 \\
\hline & $(0.001)$ & $(0.053)$ & $(0.001)$ & $(0.081)$ & $(0.000)$ & $(0.046)$ & $(0.001)$ & $(0.074)$ \\
\hline
\end{tabular}

Table 1: REs of TBRSS and DRTBRSS vs SRS in estimating population mean for $\mathrm{m}=\mathbf{5 , 6}$ 
Table 2: $\quad$ REs of TBRSS and DRTBRSS vs SRS in estimating population mean for $\mathrm{m}=7$

\begin{tabular}{lcccccc}
\hline & \multicolumn{7}{c}{$\mathrm{m}=7$} \\
\hline \multirow{2}{*}{ Distribution } & $\mathrm{RSS}$ & $\mathrm{TBRSS}$ & $\mathrm{TBRSS}$ & $\mathrm{DRSS}$ & DRTBRSS & DRTBRSS \\
\cline { 2 - 7 } Uniform(0,1) & 3.9805 & 4.5041 & 5.8385 & 8.8077 & 8.6027 & 10.8710 \\
& $(0.000)$ & $(0.001)$ & $(0.002)$ & $(0.000)$ & $(0.001)$ & $(0.003)$ \\
Normal$(0,1)$ & 3.5298 & 3.1596 & 2.7562 & 6.3801 & 11.3620 & 18.2154 \\
& $(0.000)$ & $(0.001)$ & $(0.001)$ & $(0.000)$ & $(0.000)$ & $(0.002)$ \\
Logistic(0,1) & 3.2446 & 2.6555 & 2.0394 & 5.2040 & 12.7913 & 22.4060 \\
& $(0.000)$ & $(0.001)$ & $(0.002)$ & $(0.000)$ & $(0.001)$ & $(0.004)$ \\
Exponential(1) & 2.6809 & 1.6487 & 0.8100 & 4.0078 & 2.9570 & 1.7326 \\
& $(0.001)$ & $(0.119)$ & $(0.282)$ & $(0.001)$ & $(0.191)$ & $(0.277)$ \\
Weibull(1,3) & 2.7094 & 1.6482 & 0.8167 & 3.9485 & 2.9634 & 1.7410 \\
& $(0.002)$ & $(0.362)$ & $(0.846)$ & $(0.002)$ & $(0.575)$ & $(0.829)$ \\
Gamma(2,3) & 2.7225 & 1.6504 & 0.7992 & 4.9011 & 4.5504 & 2.8714 \\
& $(0.000)$ & $(0.039)$ & $(0.094)$ & $(0.000)$ & $(0.067)$ & $(0.097)$ \\
\hline
\end{tabular}

The following remarks based on Tables1-2:

1. A substantial gain in efficiency is obtained using DRTBRSS for estimating population mean.

2. DRTBRSS is more efficient than TBRSS for all values of $\mathrm{k}$.

3. When underlying distribution is asymmetric, DRTBRSS remains at least efficient than TBRSS and SRS for all values of $\mathrm{k}$. This supports robustness of the proposed mean estimator.

4. DRTBRSS is also more efficient than RSS for $\mathrm{k}=2$.

5. For asymmetric distributions, biasedness increases as k gets large with minimum biasness occurs at $\mathrm{k}=0,1$.

\section{Estimation of population median}

Median is reliable measure of center tendency when underlying distribution is asymmetric or highly skewed. We define median estimators based on SRS, RSS, TBRSS and DRTBRSS. An extensive simulation study is also conducted to compare the efficiency of the median estimators based on RSS, TBRSS and DRTBRSS relative to conventional estimator based on SRS. Let $X_{1}, X_{2}, X_{3}, \ldots, X_{m}$ be a SRS of size $m$. Then, the SRS estimator of population median, say $\theta$, is defined as

$$
\hat{\theta}_{\mathrm{SRS}}= \begin{cases}\mathrm{X}_{((\mathrm{m}+1) / 2: \mathrm{m})} & \text { if } \mathrm{m} \text { is odd } \\ \frac{X_{((\mathrm{m} / 2): \mathrm{m})}+\mathrm{X}_{(((\mathrm{m}+2) / 2): \mathrm{m})}}{2} & \text { if } \mathrm{m} \text { is even }\end{cases}
$$


Suppose the measured RSS units are $X_{1(1: m)}, X_{2(2: m)}, \ldots, X_{m(m: m)}$. Then, the RSS estimator of population median is given by

$$
\hat{\theta}_{\mathrm{RSS}}=\operatorname{Median}\left\{\mathrm{X}_{\mathrm{i}(\mathrm{i}: \mathrm{m})} \quad \mathrm{i}=1,2, \ldots, \mathrm{m}\right.
$$

The population median estimator under TBRSS is defined by

$$
\hat{\theta}_{\text {TBRSS }}=\operatorname{Median} \begin{cases}X_{\mathrm{i}(1 \mathrm{k})} & \mathrm{i}=1,2, \ldots, \mathrm{k} \\ X_{\mathrm{i}(\mathrm{i}: \mathrm{m}-\mathrm{k})} & \mathrm{i}=\mathrm{k}+1, \mathrm{k}+2, \ldots, \mathrm{m}-2 \mathrm{k} \\ X_{\mathrm{i}((\mathrm{m}: \mathrm{m})} & \mathrm{i}=\mathrm{m}-2 \mathrm{k}+1, \ldots, \mathrm{m}\end{cases}
$$

Similarly, Let $\mathrm{X}_{1(\mathrm{k}: \mathrm{m})}^{\cdot}, \mathrm{X}_{2(\mathrm{k}: \mathrm{m})}^{\cdot}, \ldots, \mathrm{X}_{\mathrm{k}(\mathrm{k}: \mathrm{m})}^{\cdot}, \mathrm{X}_{(\mathrm{k}+1)(\mathrm{k}+1) \mathrm{m})}^{\cdot}, \ldots, \mathrm{X}_{(\mathrm{m}-\mathrm{k})(\mathrm{m}-\mathrm{k}: \mathrm{m})}, \mathrm{X}_{(\mathrm{m}-\mathrm{k}+1)(\mathrm{m}-\mathrm{k}+1): \mathrm{m})}^{\cdot}, \ldots$, $\mathrm{X}_{\mathrm{m}(\mathrm{m}-\mathrm{k}+1 \mathrm{~m})}$ be DRTBRSS of size $\mathrm{m}$. Then, the median estimator of population median is defined as

$$
\hat{\theta}_{\text {DRTBRSS }}=\operatorname{Median} \begin{cases}X_{i(\mathrm{k}: \mathrm{m})}^{\cdot} & \mathrm{i}=1,2, \ldots, \mathrm{k} \\ \mathrm{X}_{\mathrm{i}(\mathrm{i}: \mathrm{m})}^{\cdot} & \mathrm{i}=\mathrm{k}+1, \mathrm{k}+2, \ldots, \mathrm{m}-\mathrm{k} \\ \mathrm{X}_{\mathrm{i}(\mathrm{m}-\mathrm{k}+\mathrm{l}: \mathrm{m})} & \mathrm{i}=\mathrm{m}-\mathrm{k}+1, \mathrm{~m}-\mathrm{k}+2, \ldots, \mathrm{m}\end{cases}
$$

The REs of the above median estimators with respect to $\hat{\theta}_{\mathrm{SRS}}$ are defined as

$$
\operatorname{eff}\left(\hat{\theta}_{\mathrm{J}}, \hat{\theta}_{\mathrm{SRS}}\right)=\frac{\operatorname{MSE}\left(\hat{\theta}_{\mathrm{SRS}}\right)}{\operatorname{MSE}\left(\hat{\theta}_{\mathrm{J}}\right)} \mathrm{J}=\mathrm{TBRSS}, \operatorname{DRTBRSS}
$$

REs of the median estimators are calculated using $\mathrm{Eq}(12)$ for different sample sizes from

\begin{tabular}{|c|c|c|c|c|c|c|c|c|}
\hline \multirow[b]{3}{*}{ Distribution } & \multicolumn{4}{|c|}{$\mathrm{m}=5$} & \multicolumn{4}{|c|}{$\mathrm{m}=6$} \\
\hline & RSS & TBRSS & DRSS & DRTBRSS & RSS & TBRSS & DRSS & DRTBRSS \\
\hline & $\mathrm{k}=0,1$ & $\mathrm{k}=2$ & $\mathrm{k}=0,1$ & $\mathrm{k}=2$ & $\mathrm{k}=0,1$ & $\mathrm{k}=2$ & $\mathrm{k}=0,1$ & $\mathrm{k}=2$ \\
\hline Uniform $(0,1)$ & $\begin{array}{l}1.8778 \\
(0.001)\end{array}$ & $\begin{array}{l}1.4129 \\
(0.002)\end{array}$ & $\begin{array}{l}2.8297 \\
(0.001)\end{array}$ & $\begin{array}{l}4.7655 \\
(0.045)\end{array}$ & $\begin{array}{l}2.4304 \\
(0.000)\end{array}$ & $\begin{array}{l}2.1036 \\
(0.000)\end{array}$ & $\begin{array}{l}4.4992 \\
(0.001)\end{array}$ & $\begin{array}{l}6.5409 \\
(0.001)\end{array}$ \\
\hline $\operatorname{Normal}(0,1)$ & $\begin{array}{l}2.0775 \\
(0.003)\end{array}$ & $\begin{array}{l}1.5330 \\
(0.003)\end{array}$ & $\begin{array}{l}3.3636 \\
(0.002)\end{array}$ & $\begin{array}{l}5.6215 \\
(0.117)\end{array}$ & $\begin{array}{l}2.7458 \\
(0.001)\end{array}$ & $\begin{array}{l}2.2223 \\
(0.000)\end{array}$ & $\begin{array}{l}4.9685 \\
(0.002)\end{array}$ & $\begin{array}{l}7.7842 \\
(0.001)\end{array}$ \\
\hline $\operatorname{Logistic}(0,1)$ & $\begin{array}{c}2.184 \\
(0.003)\end{array}$ & $\begin{array}{l}1.5555 \\
(0.004)\end{array}$ & $\begin{array}{l}3.6035 \\
(0.003)\end{array}$ & $\begin{array}{l}5.9365 \\
(0.190)\end{array}$ & $\begin{array}{l}2.8337 \\
(0.004)\end{array}$ & $\begin{array}{l}2.3288 \\
(0.004)\end{array}$ & $\begin{array}{l}4.4336 \\
(0.001)\end{array}$ & $\begin{array}{l}8.2111 \\
(0.003)\end{array}$ \\
\hline Exponential(1) & $\begin{array}{l}2.3378 \\
(0.045)\end{array}$ & $\begin{array}{l}1.5957 \\
(0.062)\end{array}$ & $\begin{array}{l}3.7591 \\
(0.029)\end{array}$ & $\begin{array}{l}8.7077 \\
(0.067)\end{array}$ & $\begin{array}{l}2.8789 \\
(0.048)\end{array}$ & $\begin{array}{l}2.3091 \\
(0.065)\end{array}$ & $\begin{array}{l}5.5119 \\
(0.036)\end{array}$ & $\begin{array}{l}8.6203 \\
(0.019)\end{array}$ \\
\hline Weibull $(1,3)$ & $\begin{array}{l}2.3186 \\
(0.122)\end{array}$ & $\begin{array}{l}1.5906 \\
(0.184)\end{array}$ & $\begin{array}{l}3.8483 \\
(0.083)\end{array}$ & $\begin{array}{l}8.9325 \\
(0.205)\end{array}$ & $\begin{array}{l}2.8706 \\
(0.146)\end{array}$ & $\begin{array}{l}2.2653 \\
(0.199)\end{array}$ & $\begin{array}{l}5.4210 \\
(0.104)\end{array}$ & $\begin{array}{l}8.5390 \\
(0.062)\end{array}$ \\
\hline $\operatorname{Gamma}(2,3)$ & $\begin{array}{l}2.2865 \\
(0.014)\end{array}$ & $\begin{array}{l}1.5967 \\
(0.021)\end{array}$ & $\begin{array}{l}3.4832 \\
(0.010)\end{array}$ & $\begin{array}{r}7.4066 \\
(0.040)\end{array}$ & $\begin{array}{l}2.2900 \\
(0.016)\end{array}$ & $\begin{array}{l}2.2356 \\
(0.022)\end{array}$ & $\begin{array}{l}5.3478 \\
(0.012)\end{array}$ & $\begin{array}{l}8.0653 \\
(0.008)\end{array}$ \\
\hline
\end{tabular}
both symmetric and asymmetric distributions and reported in Tables 3-4.

Table 3: REs of TBRSS and DRTBRSS vs SRS in estimating population median for $m=5,6$ 
Table 4: REs of TBRSS and DRTBRSS vs SRS in estimating population median for $m=7$

\begin{tabular}{ccccccc}
\hline & \multicolumn{7}{c}{$\mathrm{m}=7$} \\
\hline \multirow{2}{*}{ Distribution } & $\mathrm{RSS}$ & $\mathrm{TBRSS}$ & $\mathrm{TBRSS}$ & $\mathrm{DRSS}$ & DRTBRSS & DRTBRSS \\
\cline { 2 - 7 } & $\mathrm{k}=0,1$ & $\mathrm{k}=2$ & $\mathrm{k}=3$ & $\mathrm{k}=0,1$ & $\mathrm{k}=2$ & $\mathrm{k}=3$ \\
\hline Uniform(0,1) & 2.2164 & 1.9441 & 1.2532 & 3.7198 & 4.8847 & 12.2802 \\
& $(0.001)$ & $(0.001)$ & $(0.000)$ & $(0.000)$ & $(0.001)$ & $(0.000)$ \\
Normal$(0,1)$ & 2.5118 & 2.1181 & 1.3169 & 4.2633 & 5.7649 & 14.6161 \\
& $(0.002)$ & $(0.001)$ & $(0.001)$ & $(0.001)$ & $(0.001)$ & $(0.002)$ \\
Logistic(0,1) & 2.5905 & 2.2001 & 1.3184 & 4.5161 & 6.0171 & 15.2504 \\
& $(0.000)$ & $(0.002)$ & $(0.002)$ & $(0.003)$ & $(0.002)$ & $(0.000)$ \\
Exponential(1) & 2.7723 & 2.3210 & 1.3572 & 4.7733 & 6.4496 & 16.3636 \\
& $(0.027)$ & $(0.033)$ & $(0.051)$ & $(0.015)$ & $(0.012)$ & $(0.005)$ \\
Weibull(1,3) & 2.7156 & 2.2585 & 1.3624 & 4.7319 & 6.3991 & 16.4053 \\
& $(0.086)$ & $(0.098)$ & $(0.147)$ & $(0.046)$ & $(0.043)$ & $(0.012)$ \\
Gamma(2,3) & 2.6290 & 2.1847 & 1.3215 & 4.4769 & 6.0733 & 15.2879 \\
& $(0.010)$ & $(0.011)$ & $(0.019)$ & $(0.006)$ & $(0.005)$ & $(0.002)$ \\
\hline
\end{tabular}

Tables3-4 describe the following:

1. DRTBRSS is more efficient than TBRSS and RSS for estimating population median.

2. The efficiency of DRTBRSS increases as $\mathrm{k}$ gets large for all considered sample sizes. For example, for $m=7$ and $k=2$, the efficiency of DRTBRSS is 4.8847 and it is 12.2802 for $\mathrm{k}=3$ in estimating median of standard uniform distribution.

3. Biasedness also decreases as $m$ gets large.

\section{Ranking with auxiliary variable}

In many practical problems the variable of interest, $\mathrm{Y}$, is hard to measure and difficult to rank as well but a auxiliary variable, $\mathrm{X}$, correlated with, $\mathrm{Y}$, can easily be measured. Then the auxiliary variable can be used for the ranking of the sampling units. For instance, the assessment of the status of bomb blast sites is usually costly. But, often, a great deal of knowledge about bomb blast sites can be obtained from video, photos etc. and then be used to rank the bomb blast sites. In this section, we follow Stokes (1977) idea in which ranking is performed using auxiliary variable,say $\mathrm{X}$, that can be measured easily. Stokes (1977) proposed the following model with the assumptions (1) the regression of $Y$ on $X$ is linear (2) the underlying distributions of standardized variables $\frac{Y-\mu_{Y}}{\sigma_{Y}}$ and $\frac{X-\mu_{X}}{\sigma_{X}}$ are same.

$$
Y_{i[i: m] j}=\mu_{X}+\rho \frac{\sigma_{X}}{\sigma_{Y}}\left(X_{i(i: m) j}-\mu_{Y}\right)+\varepsilon_{i j}, i=1,2, \ldots, m ; j=1,2, \ldots, r
$$


Here, $\mathrm{X}_{\mathrm{i}(\mathrm{i}: \mathrm{m})}$ and $\varepsilon$ are independent and $\varepsilon$ has mean zero and variance $\sigma_{\varepsilon}^{2}=\sigma_{\mathrm{Y}}^{2}\left(1-\rho^{2}\right)$. Let $\mathrm{Y}_{\mathrm{i}[\mathrm{i}: \mathrm{m}] \mathrm{j}}$ is the ith smallest value of $\mathrm{Y}$ corresponding to ith smallest value of $\mathrm{X}$ i.e. $\mathrm{X}_{\mathrm{i}(\mathrm{i}: \mathrm{m}) \mathrm{j}}$ in jth replication.

\subsection{Estimation using auxiliary variable}

Let $\left(\mathrm{Y}_{\mathrm{i}}, \mathrm{X}_{\mathrm{i}}\right), \mathrm{i}=1,2,3, \ldots, \mathrm{m}$ be the bivariate $\mathrm{SRS}$ from bivariate normal distribution. The RSS estimator of population mean using auxiliary variable $X$ i.e. $\bar{Y}_{\mathrm{RSSA}}$ is defined as

$$
\overline{\mathrm{Y}}_{\mathrm{RSSA}}=\frac{1}{\mathrm{mr}} \sum_{\mathrm{j}=\mathrm{li}=1}^{\mathrm{r}} \sum_{\mathrm{i}[1: \mathrm{m}] \mathrm{j}}
$$

with variance

$$
\operatorname{Var}\left(\overline{\mathrm{Y}}_{\mathrm{RSSA}}\right)=\frac{1}{\mathrm{~m}^{2} \mathrm{r}}\left\{m \sigma_{\mathrm{Y}}^{2}\left(1-\rho^{2}\right)+\rho^{2} \frac{\sigma_{\mathrm{Y}}^{2}}{\sigma_{\mathrm{X}}^{2}} \sum_{\mathrm{i}=1}^{\mathrm{m}} \sigma_{(\mathrm{i}: \mathrm{m})}^{2}\right\}
$$

Similarly, the TBRSSA estimator of population mean is given by

$$
\overline{\mathrm{Y}}_{\mathrm{TBRSSA}}=\frac{1}{\mathrm{mr}}\left(\sum_{\mathrm{j}=1 \mathrm{i}=1}^{\mathrm{r}} \sum_{\mathrm{i}[1: \mathrm{m}] \mathrm{j}}^{\mathrm{k}}+\sum_{\mathrm{j}=1 \mathrm{i}=\mathrm{k}+1}^{\mathrm{r}} \sum_{\mathrm{i}-\mathrm{k}}^{\mathrm{m}} \mathrm{Y}_{\mathrm{i}: \mathrm{m}] \mathrm{j}}+\sum_{\mathrm{j}=1 \mathrm{i}=(\mathrm{m}-\mathrm{k}+1}^{\mathrm{r}} \sum_{\mathrm{i}[\mathrm{m}: \mathrm{m}] \mathrm{j}}^{\mathrm{m}}\right)
$$

with variance

$$
\operatorname{Var}\left(\overline{\mathrm{Y}}_{\mathrm{TBRSSA}}\right)=\frac{1}{\mathrm{~m}^{2} \mathrm{r}}\left\{m \sigma_{\mathrm{Y}}^{2}\left(1-\rho^{2}\right)+\rho^{2} \frac{\sigma_{\mathrm{Y}}^{2}}{\sigma_{\mathrm{X}}^{2}}\left(2 \mathrm{k} \sigma_{(1: \mathrm{m})}^{2}+\sum_{\mathrm{i}=\mathrm{k}+1}^{\mathrm{m}-\mathrm{k}} \sigma_{(\mathrm{i}: \mathrm{m})}^{2}\right)\right\}
$$

Analogous to estimators (14) and (16), the mean estimator under DRTBRSSA is given by

$$
\overline{\mathrm{Y}}_{\text {DRTBRSSA }}=\frac{1}{\mathrm{mr}}\left(\sum_{\mathrm{j}=1 \mathrm{i}=1}^{\mathrm{r}} \sum^{\mathrm{k}} \mathrm{Y}_{\mathrm{i}[\mathrm{k}: \mathrm{m}] \mathrm{j}}+\sum_{\mathrm{j}=1 \mathrm{i}=\mathrm{k}+1}^{\mathrm{r}} \sum_{\mathrm{i}-\mathrm{i}: \mathrm{m}] \mathrm{j}}^{\mathrm{m}}+\sum_{\mathrm{j}=1 \mathrm{i}=(\mathrm{m}-\mathrm{k}+1}^{\mathrm{r}} \mathrm{Y}_{\mathrm{i}[\mathrm{m}-\mathrm{k}+1: \mathrm{m}] \mathrm{j}}^{\mathrm{m}}\right)
$$

It is easy to show that the proposed estimator is unbiased estimator of population mean and it has variance given by

$$
\operatorname{Var}\left(\overline{\mathrm{Y}}_{\text {DRTBRSSA }}\right)=\frac{1}{\mathrm{~m}^{2} \mathrm{r}}\left\{m \sigma_{\mathrm{Y}}^{2}\left(1-\rho^{2}\right)+\rho^{2} \frac{\sigma_{\mathrm{Y}}^{2}}{\sigma_{\mathrm{X}}^{2}}\left(2 \mathrm{k} \sigma_{(\mathrm{k}: \mathrm{m})}^{2 \cdot}+\sum_{\mathrm{i}=\mathrm{k}+1}^{\mathrm{m}-\mathrm{k}} \sigma_{(\mathrm{i}: \mathrm{m})}^{2 \cdot}\right)\right\}
$$

The RE of $\overline{\mathrm{Y}}_{\mathrm{RSSA}}$ with respect to $\overline{\mathrm{Y}}_{\mathrm{SRS}}$ is given by

$$
\operatorname{RE}\left(\overline{\mathrm{Y}}_{\mathrm{RSSA}}, \overline{\mathrm{Y}}_{\mathrm{SRS}}\right)=\frac{\mathrm{m} \sigma_{\mathrm{X}}^{2}}{\mathrm{~m} \sigma_{\mathrm{X}}^{2}\left(1-\rho^{2}\right)+\rho^{2} \sum_{\mathrm{i}=1}^{\mathrm{m}} \sigma_{(\mathrm{i}: \mathrm{m})}^{2}}
$$

The RE of $\overline{\mathrm{Y}}_{\mathrm{TBRSSA}}$ with respect to $\overline{\mathrm{Y}}_{\mathrm{SRS}}$ is given by

$$
\operatorname{RE}\left(\bar{Y}_{\text {TBRSSA }}, \bar{Y}_{\text {SRS }}\right)=\frac{m \sigma_{X}^{2}}{m \sigma_{X}^{2}\left(1-\rho^{2}\right)+\rho^{2}\left(2 \mathrm{k} \sigma_{(1: m)}^{2}+\sum_{i=k+1}^{m-k} \sigma_{(i: m)}^{2}\right)}
$$


The RE of the proposed estimator $\overline{\mathrm{Y}}_{\mathrm{DRTBRSSA}}$ with respect to $\overline{\mathrm{Y}}_{\mathrm{SRS}}$ is given by

$$
\operatorname{RE}\left(\overline{\mathrm{Y}}_{\text {DRTBRSSA}}, \overline{\mathrm{Y}}_{\mathrm{SRS}}\right)=\frac{\mathrm{m} \sigma_{\mathrm{X}}^{2}}{\mathrm{~m} \sigma_{\mathrm{X}}^{2}\left(1-\rho^{2}\right)+\rho^{2}\left(2 \mathrm{k} \sigma_{(\mathrm{k}: \mathrm{m})}^{2 \cdot}+\sum_{\mathrm{i}=\mathrm{k}+1}^{\mathrm{m}-\mathrm{k}} \sigma_{(\mathrm{i}: \mathrm{m})}^{2 \cdot}\right)}
$$

In case of median estimation, the REs of the median estimators $\hat{\theta}_{\mathrm{JA}}$ with respect to $\hat{\theta}_{\mathrm{SRS}}$ is defined as

$$
\operatorname{eff}\left(\hat{\theta}_{\mathrm{JA}}, \hat{\theta}_{\mathrm{SRS}}\right)=\frac{\operatorname{MSE}\left(\hat{\theta}_{\mathrm{SRS}}\right)}{\operatorname{MSE}\left(\hat{\theta}_{\mathrm{JA}}\right)} \mathrm{J}=\mathrm{TBRSS}, \operatorname{DRTBRSS}
$$

The REs of mean and median estimators, when ranking based on auxiliary variable, are calculated for different sample sizes from standard bivariate normal distribution and reported in Tables5-6. As expected, the performance of the estimators depend on value of correlation coefficient. The estimators become more precise as correlation increases and vice-versa. However, performance of the proposed mean and median estimators under both perfect and imperfect rankings dominates as depicted in Tables5-6. For instance, if $\rho= \pm 0.25$ and $m=5$, the REs of mean and median estimators based on TBRSS are 1.0465 and 1.0112 respectively, while these are 3.4180 and 2.8000 under DRTBRSS. This shows robustness of the proposed mean and median estimators under imperfect rankings.

Table 5: REs of TBRSS and DRTBRSS vs SRS in estimating population mean

\begin{tabular}{|c|c|c|c|c|c|c|c|}
\hline \multirow[b]{2}{*}{$\begin{array}{l}\text { Sample } \\
\text { size }\end{array}$} & \multirow[b]{2}{*}{$\rho$} & RSS & TBRSS & TBRSS & DRSS & DRTBRSS & DRTBRSS \\
\hline & & $\mathrm{k}=0,1$ & $\mathrm{k}=2$ & - & $\mathrm{k}=0,1$ & $\mathrm{k}=2$ & - \\
\hline \multirow[t]{4}{*}{$\mathrm{m}=5$} & \pm 0.25 & 1.0203 & 1.0465 & - & 2.8207 & 3.4180 & - \\
\hline & \pm 0.50 & 1.2049 & 1.1634 & - & 2.9646 & 3.9133 & - \\
\hline & +075 & 1.5767 & 1.5324 & - & 3.3862 & 4.9617 & - \\
\hline & \pm 0.90 & 2.0695 & 1.9248 & - & 3.8677 & 6.4791 & - \\
\hline \multirow[t]{4}{*}{$\mathrm{m}=6$} & \pm 0.25 & 1.0463 & 1.0488 & - & 3.2525 & 3.8583 & - \\
\hline & \pm 0.50 & 1.1990 & 1.1988 & - & 3.4825 & 4.3209 & - \\
\hline & +075 & 1.6290 & 1.5754 & - & 3.9543 & 5.6212 & - \\
\hline & \pm 0.90 & 2.2308 & 2.0804 & - & 4.4867 & 7.3167 & - \\
\hline \multirow[t]{4}{*}{$\mathrm{m}=7$} & \pm 0.25 & 1.0667 & 1.0446 & 1.0422 & 3.5602 & 4.4316 & 4.7670 \\
\hline & \pm 0.50 & 1.2168 & 1.2161 & 1.1559 & 3.8060 & 4.8675 & 5.7171 \\
\hline & +075 & 1.6805 & 1.6501 & 1.5221 & 4.5700 & 6.2275 & 7.9375 \\
\hline & \pm 0.90 & 2.3805 & 2.2575 & 2.0888 & 5.4541 & 8.2667 & 11.3578 \\
\hline
\end{tabular}
using bivariate normal distribution. 
Table 6: REs of TBRSS and DRTBRSS vs SRS in estimating population median using bivariate normal distribution.

\begin{tabular}{|c|c|c|c|c|c|c|c|}
\hline \multirow[b]{2}{*}{$\begin{array}{l}\text { Sample } \\
\text { size }\end{array}$} & \multirow[b]{2}{*}{$\rho$} & RSS & TBRSS & TBRSS & DRSS & DRTBRSS & DRTBRSS \\
\hline & & $\mathrm{k}=0,1$ & $\mathrm{k}=2$ & - & $\mathrm{k}=0,1$ & $\mathrm{k}=2$ & - \\
\hline \multirow[t]{4}{*}{$m=5$} & \pm 0.25 & 1.0163 & 1.0112 & - & 2.1104 & 2.8000 & - \\
\hline & \pm 0.50 & 1.1050 & 1.0613 & - & 2.2457 & 3.1718 & - \\
\hline & \pm 0.75 & 1.3729 & 1.1478 & - & 2.4903 & 4.0071 & - \\
\hline & \pm 0.90 & 1.6361 & 1.2756 & - & 2.8688 & 5.2314 & - \\
\hline \multirow[t]{4}{*}{$m=6$} & \pm 0.25 & 1.0317 & 1.0583 & - & 2.6860 & 3.3593 & - \\
\hline & \pm 0.50 & 1.1424 & 1.1075 & - & 2.9646 & 3.6768 & - \\
\hline & \pm 0.75 & 1.4446 & 1.3492 & - & 3.4836 & 4.6870 & - \\
\hline & \pm 0.90 & 2.0084 & 1.6645 & - & 4.1399 & 6.1109 & - \\
\hline \multirow[t]{4}{*}{$m=7$} & \pm 0.25 & 1.0344 & 1.0258 & 1.0136 & 2.5543 & 2.9870 & 4.1106 \\
\hline & \pm 0.50 & 1.1404 & 1.0811 & 1.0165 & 2.7033 & 3.2885 & 4.8828 \\
\hline & \pm 0.75 & 1.3910 & 1.2427 & 1.0241 & 3.0981 & 4.0540 & 6.7677 \\
\hline & \pm 0.90 & 1.8050 & 1.5627 & 1.0801 & 3.5981 & 4.8352 & 9.7443 \\
\hline
\end{tabular}

\section{Illustration with real data}

In this section we use a real data set to illustrate the efficiency of the proposed MBRSS and TBRSS schemes with respect to SRS in estimating mean and median height of 399 conifer trees. The data based on two variables: $X$, the diameter in centimeters at breast height, and $Y$, the entire height in feet, for more detail see Platt et al. (1988). The summary statistics of the two variables are given by

$$
\begin{aligned}
& \mu_{X}=\frac{1}{399} \sum_{i=1}^{399} X_{i}=21.09, \sigma_{X}^{2}=\frac{1}{399} \sum_{i=1}^{399}\left(x_{i}-\mu_{X}\right)^{2}=329.785, \operatorname{Med}_{X}=14.5, \text { Skewness }_{X}=1.05 \\
& \mu_{Y}=\frac{1}{399} \sum_{i=1}^{399} Y_{i}=52.34, \sigma_{Y}^{2}=\frac{1}{399} \sum_{i=1}^{399}\left(y_{i}-\mu_{Y}\right)^{2}=3262.6944, \operatorname{Med}_{Y}=29, \text { Skewness }_{Y}=1.63 \\
& \rho=0.876
\end{aligned}
$$

\begin{tabular}{|c|c|c|c|c|c|c|c|}
\hline \multirow[b]{2}{*}{$\begin{array}{l}\text { Sample } \\
\text { size }\end{array}$} & \multirow[b]{2}{*}{ Ranking } & RSS & TBRSS & TBRSS & DRSS & DRTBRSS & DRTBRSS \\
\hline & & $\mathrm{k}=0,1$ & $\mathrm{k}=2$ & - & $\mathrm{k}=0,1$ & $\mathrm{k}=2$ & - \\
\hline \multirow[t]{2}{*}{$m=5$} & Imperfect & 1.9872 & 1.4000 & - & 3.0853 & 1.8023 & - \\
\hline & Perfect & 2.2975 & 1.4217 & - & 3.2575 & 1.8113 & - \\
\hline \multirow[t]{2}{*}{$m=6$} & Imperfect & 2.1280 & 1.6404 & - & 3.6247 & 1.8531 & - \\
\hline & Perfect & 2.6083 & 1.6459 & - & 4.0559 & 1.8553 & - \\
\hline \multirow[t]{2}{*}{$\mathrm{m}=7$} & Imperfect & 2.2985 & 1.8594 & 1.0078 & 4.1961 & 2.0922 & 1.0088 \\
\hline & Perfect & 2.9367 & 1.9298 & 1.0172 & 4.7693 & 2.1046 & 1.0190 \\
\hline
\end{tabular}

Table 7: $\quad$ REs of TBRSS and DRTBRSS vs SRS in estimating mean height of 399 trees (Y) under perfect and imperfect rankings 
Table 8: REs of TBRSS and DRTBRSS vs SRS in estimating median height of 399 trees (Y) under perfect and imperfect rankings

\begin{tabular}{|c|c|c|c|c|c|c|c|}
\hline \multirow[b]{2}{*}{$\begin{array}{l}\text { Sample } \\
\text { size }\end{array}$} & \multirow[b]{2}{*}{ Ranking } & RSS & TBRSS & TBRSS & DRSS & DRTBRSS & DRTBRSS \\
\hline & & $\mathrm{k}=0,1$ & $\mathrm{k}=2$ & - & $\mathrm{k}=0,1$ & $\mathrm{k}=2$ & - \\
\hline \multirow[t]{2}{*}{$m=5$} & Imperfect & 2.6004 & 1.6387 & - & 5.7513 & 15.2948 & - \\
\hline & Perfect & 3.3216 & 1.8724 & - & 6.3082 & 17.5336 & - \\
\hline \multirow[t]{2}{*}{$m=6$} & Imperfect & 3.0695 & 2.0631 & - & 6.6287 & 15.0117 & - \\
\hline & Perfect & 3.6886 & 2.4811 & - & 7.4049 & 17.1767 & - \\
\hline \multirow[t]{2}{*}{$\mathrm{m}=7$} & Imperfect & 3.5451 & 2.8232 & 1.3436 & 8.7427 & 12.6677 & 29.2857 \\
\hline & Perfect & 4.3411 & 3.3419 & 1.5022 & 9.3923 & 13.8288 & 35.2581 \\
\hline
\end{tabular}

Since both variables have non-zero skewness so data is asymmetrically distributed. REs of mean and median estimators for different sample sizes are reported in Tables7-8. These tables support robustness of mean and median estimators based on DRTBRSS design under both perfect and imperfect rankings relative to TBRSS. There is decay in the efficiency of mean and median estimators under imperfect rankings as it expected due to error in rankings. But the REs of estimators based on DRTBRSS give efficient estimates as compare to TBRSS. RE of the median estimator based on proposed DRTBRSS outperforms relative to ordinary RSS and TBRSS. For instance, REs of the median estimators based on RSS and TBRSS, under imperfect rankings for $m=5$, are 2.6004 and 1.6387 respectively. While, it is 15.2948 in case of DRTBRSS.

\section{Conclusion}

In this paper, we suggested DRTBRSS scheme for estimating population mean and median. It is observed that when underlying distribution is symmetric, the population mean estimator based on DRTBRSS is unbiased and more efficient than the estimators based on SRS, RSS and TBRSS for all values of $\mathrm{k}$. As regards population median estimation, the proposed sampling design outperforms among others considered sampling designs. The performance of the mean and median estimators under imperfect rankings is also studied. It is found that the DRTBRSS scheme is an efficient alternative to the existing SRS, RSS and TBRSS schemes for estimating the mean and median of symmetric population. The larger efficiency of the proposed estimators relative to TBRSS estimators, under asymmetric populations, also supports its robustness. However, biasness increases as $\mathrm{k}$ gets large when underlying population is asymmetric. Therefore, it is recommended to use DRTBRSS in estimating population mean and median of symmetric population. It can also be used to estimate mean and median of asymmetric population subject to a compromise on biasness.

\section{Acknowledgement:}

The first author is thankful to the unknown referees for their encouragement and valuable suggestions to bring the article in the present form. 


\section{References}

1. Al-Nasser, A. D. (2007). L-ranked set sampling: A generalization procedure for robust visual sampling. Communications in Statistics- Simulation and Computation 36 (1), 33-43.

2. Al-Omari, A. I. (2011). Estimation of mean based on modified robust extreme ranked set sampling. Journal of Statistical Computation and Simulation 81 (8), 1055-1066.

3. Al-Omari, A. I. and M. Z. Raqab (2013). Estimation of the population mean and median using truncation-based ranked set samples. Journal of Statistical Computation and Simulation 83 (8), 1453-1471.

4. Al-Saleh, M. F. and M. A. Al-Kadiri (2000). Double-ranked set sampling. Statistics \& Probability Letters 48 (2), 205-212.

5. David, H. and H. Nagaraja (2003). Order statistics, John Wiley \& Sons. Inc., New York.

6. Dell, T. and J. Clutter (1972). Ranked set sampling theory with order statistics background. Bio-metrics, 545-555.

7. Stokes, S. L (1977). Ranked set sampling with concomitant variables. Communications in Statistics-Theory and Methods 6 (12), 1207-1211.

8. McIntyre, G. (1952). A method for unbiased selective sampling, using ranked sets. Crop and Pasture Science 3 (4), 385-390.

9. Platt, W. J., G. W. Evans, and S. L. Rathbun (1988). The population dynamics of a long-lived conifer (pinus palustris). American Naturalist, 491-525.

10. Samawi, H. M., M. S. Ahmed, and W. Abu-Dayyeh (1996). Estimating the population mean using extreme ranked set sampling. Biometrical Journal 38 (5), 577-586.

11. Stokes, S. L. (1980). Estimation of variance using judgment ordered ranked set samples. Biometrics, 35-42.

12. Zamanzade, E. and Al-Omari, A. I (2016). New ranked set sampling for estimating the population mean and variance. Hacettepe Journal of Mathematics and Statistics, 1891. 